\title{
Bifidobacterium bifidum R0071 results in a greater proportion of healthy days and a lower percentage of academically stressed students reporting a day of cold/flu: a randomised, double-blind, placebo-controlled study
}

\author{
Bobbi Langkamp-Henken ${ }^{1 *}$, Cassie C. Rowe ${ }^{1}$, Amanda L. Ford $^{1}$, Mary C. Christman $^{2,3,4}$, \\ Carmelo Nieves $\mathrm{Jr}^{1}$, Lauren Khouri ${ }^{1}$, Gretchen J. Specht ${ }^{1}$, Stephanie-Anne Girard ${ }^{5}$, \\ Samuel J. Spaiser ${ }^{1}$ and Wendy J. Dahl ${ }^{1}$ \\ ${ }^{1}$ Food Science and Human Nutrition Department, University of Florida, 572 Newell Drive, PO Box 110370, \\ Gainesville, FL 32611-0370, USA \\ ${ }^{2}$ Department of Statistics, University of Florida, 102 Griffin-Floyd Hall, Gainesville, FL 32611, USA \\ ${ }^{3}$ Department of Biology, University of Florida, Bartram Hall, 876 Newell Drive, Gainesville, FL 32611, USA \\ ${ }^{4}$ MCC Statistical Consulting LLC, Gainesville, FL, USA \\ ${ }^{5}$ Lallemand Health Solutions, Montréal, QC, Canada
}

(Submitted 5 August 2014 - Final revision received 20 October 2014 - Accepted 30 October 2014 - First published online 21 January 2015)

\begin{abstract}
Acute psychological stress is positively associated with a cold/flu. The present randomised, double-blind, placebo-controlled study examined the effect of three potentially probiotic bacteria on the proportion of healthy days over a 6-week period in academically stressed undergraduate students ( $n$ 581) who received Lactobacillus helveticus R0052, Bifidobacterium longum ssp. infantis R0033, Bifidobacterium bifidum R0071 or placebo. On each day, participants recorded the intensity (scale: $0=$ not experiencing to $3=$ very intense) for nine cold/flu symptoms, and a sum of symptom intensity $>6$ was designated as a day of cold/flu. B. bifidum resulted in a greater proportion of healthy days than placebo $(P \leq 0.05)$. The percentage of participants reporting $\geq 1 \mathrm{~d}$ of cold/flu during the 6-week intervention period was significantly lower with $B$. bifidum than with placebo $(P<0.05)$. There were no effects of $B$. infantis or L. helveticus compared with placebo on either outcome. A predictive model accounted for influential characteristics and their interactions on daily reporting of cold/flu episodes. The proportion of participants reporting a cold on any given day was lower at weeks 2 and 3 with $B$. bifidum and B. infantis than with placebo for the average level of stress and the most commonly reported number of hours of sleep. Daily intake of bifidobacteria provides benefit related to cold/flu outcomes during acute stress.
\end{abstract}

Key words: Probiotics: Stress: Upper respiratory tract infection: Bifidobacteria: Lactobacillus belveticus

Acute psychological stress in students during academic exams is associated with decreased cellular immunity and increased intensity of cold/flu symptoms and percentage of days with a cold/flu ${ }^{(1,2)}$. Supplementation with the prebiotic galacto-oligosaccharides (GOS) resulted in lower cold/flu symptom intensity (SI) scores and a reduction in the percentage of days with a cold/flu in university students undergoing academic stress ${ }^{(1)}$. Selective fermentation of GOS resulting in increased numbers of bifidobacteria and enhanced cellmediated immunity has been proposed as the mechanism by which this prebiotic modulated immune health ${ }^{(3,4)}$. GOS produces a highly specific bifidogenic response and has not been observed in all individuals who consume this probiotic $^{(5)}$. Thus, directly providing bifidobacteria in the form of a probiotic supplement may have a bigger impact on the immune health of a population.

A recent systematic review and meta-analysis has confirmed that probiotics belonging to the genera Bifidobacterium, as well as Lactobacillus, are effective in reducing the duration of episodes of acute respiratory illness by almost a day compared with placebo in healthy children and adults ${ }^{(6)}$. None of the studies included in the meta-analysis has examined the effect of these probiotics on cold/flu in students during academic exams, a classic model for acute psychological stress ${ }^{(7)}$. A single study that has examined the effect of the combination of Lactobacillus rhamnosus LGG and

Abbreviations: B. bifidum, Bifidobacterium bifidum R0071; B. infantis, Bifidobacterium longum ssp. infantis R0033; GOS, galacto-oligosaccharides; L. helveticus, Lactobacillus belveticus R0052; SI, symptom intensity; sIgA, secretory IgA.

* Corresponding author: B. Langkamp-Henken, fax 001352392 9467, email henken@ufl.edu 
Bifidobacterium animalis ssp. lactis $\mathrm{BB}-12$ on the duration of cold/flu in college students over a 12 -week period of their spring semester was included in the meta-analysis ${ }^{(8)}$. An episode of cold/flu was defined as an affirmative answer on two consecutive days to a question asking whether the student felt sick today. The duration of the self-reported cold/flu was approximately $2 \mathrm{~d}$ shorter, and the associated symptoms were less severe with the probiotic compared with placebo. The authors suggested that the multi-species probiotic may have attenuated the inflammatory response associated with the viral infection, leading to the reduced severity and duration of the cold/flu ${ }^{(8)}$. This study, as well as a number of studies included in the meta-analysis, used multi-strain or multi-species probiotics. This may provide advantages $(9,10)$ but makes it impossible to determine which probiotic(s) in the combination was responsible for the outcomes.

The primary purpose of the present study was to examine the individual effects of Bifidobacterium bifidum R0071 (B. bifidum), Bifidobacterium longum ssp. infantis R0033 (B. infantis) and Lactobacillus helveticus R0052 (L. belveticus), probiotics shown to decrease illness episodes when provided in combination ${ }^{(11)}$, on the proportion of healthy days over a 6-week period during which undergraduate students underwent academic stress. The secondary outcomes examined participant characteristics and level of academic stress over time on the likelihood of reporting a cold on any given day. Previous studies have suggested that psychological stress and sex have an impact on gastrointestinal function, and that gastrointestinal dysfunction may contribute to additional psychological stress. In turn, stress and hours of sleep per $\mathrm{d}$ affect cold/flu outcomes ${ }^{(1,12-14)}$. Salivary secretory IgA (sIgA) was also examined as a potential biomarker of cold/flu.

\section{Participants and methods}

\section{Participants}

Participants were recruited from a large university in the state of Florida via posters, flyers, listservs, announcements and a newspaper advertisement during the 2012 autumn semester. Participants were healthy, full-time undergraduate students aged $\geq 18$ years who reported at least one cold in the past year. Potential study participants were excluded if they (1) were current smokers, (2) had chronic allergies (defined as taking daily allergy medicine), (3) did not have at least one final exam scheduled during the week of final exams, (4) did not have daily access to the Internet during the study period, (5) were unwilling to discontinue the consumption of probiotic- and prebiotic-containing foods or potentially immune-enhancing dietary supplements (i.e. prebiotics, probiotics, Echinacea, fish oil and vitamin E ( $>400 \%$ of the $\mathrm{RDA}$ or $>60 \mathrm{mg} / \mathrm{d})$ ), (6) received an immune-suppressing intervention or had an immunosuppressive illness within the last year, (7) received antibiotic therapy within 2 months of their study start date, or (8) had a cold on the 1st day of the study. All the participants completed the inclusion/exclusion questionnaire, in some cases up to 1 month before the start of the study. The present study was conducted according to the guidelines laid down in the Declaration of Helsinki, and all procedures involving human subjects were approved by the University of Florida Institutional Review Board. Written informed consent was obtained from all the participants. The present trial was registered at http://clinicaltrials.gov (identifier no. NCT01709825).

\section{Experimental design and randomisation}

Subgroups of the 581 participants started the study protocol daily over a $4 \mathrm{~d}$ period. Initially, participants completed $7 \mathrm{~d}$ of pre-baseline questionnaires (week 0) and were then randomly assigned to receive an investigational supplement (L. belveticus, B. bifidum or B. infantis) or placebo (Lallemand Health Solutions) during the 3rd week of November 2012. Participants completed daily questionnaires, consumed the study supplements and were followed for the next 6 weeks. The complete study duration was 7 weeks. Final exams for the autumn semester were held over a 1-week timespan during the 4 th or 5 th week of the intervention depending on the day each participant started on the protocol. The number and scheduled time of final exams was dependent on each participant's class schedule. On completion of final exams, participants began their winter break, which lasted for the remainder of the study.

The study was a prospective, randomised, double-blind, placebo-controlled trial. On the day of randomisation, height and weight of the participants were measured. Participants were stratified based on BMI $\left(\leq 24.9 v . \geq 25.0 \mathrm{~kg} / \mathrm{m}^{2}\right)$ and randomised to their study group using sealed envelopes. The study statistician (M. C. C.) generated the stratification and randomisation scheme using a random number generator available in $\mathrm{JMP}^{\circledR}$ Pro 10.0 (SAS Institute, Inc.), and had no direct contact with the study participants.

\section{Study capsule blinding and administration protocols}

The blinding of the study capsules was performed by the study sponsor. No one involved in the blinding had direct contact with the study participants. A total of twelve codes were randomly generated and assigned to the four interventions (three codes per intervention). On the 2nd week of the study, the supplements were provided in coded, sealed, plastic bottles that were similar in size and shape to commercially available multi-vitamin bottles. All capsules had a similar physical appearance and contained an off-white powder. Each supplement capsule contained $3 \times 10^{9}$ colony-forming units of one of the following: $L$. belveticus; $B$. bifidum; $B$. infantis (colony-forming units reflect the numbers expected after 2 years at room temperature). Placebo was composed of $3 \%$ magnesium stearate and $97 \%$ potato starch. Participants were instructed to consume one capsule per $\mathrm{d}$, with a meal, for the duration of the study. Participants were unable to determine what intervention they received throughout the study. In each group, the percentage of participants who thought they received an investigational supplement ranged from 50 to $53 \%$, which suggests successful blinding. 


\section{Online study questionnaires}

Questionnaires were administered through a Web-based survey tool (Qualtrics). Participants were sent an individual link daily to their private email address. Demographic questions were addressed on the baseline questionnaire the week before randomisation. The daily questionnaires asked students about their level of stress (scale: $0=$ no stress to $10=$ extremely stressed), consumption of the study capsules, hours of sleep, antibiotic intake, cold and flu symptoms, and SI. Completion of the questionnaires was monitored by study coordinators, who contacted the participants via email and/or phone to remind them of the approaching deadline for submission of the questionnaires. After the participants completed and submitted the questionnaires, they could no longer go back to modify their responses.

\section{Primary outcome}

Proportion of healthy days. Daily questionnaires were used to collect information related to the primary outcome (i.e. the effect of the interventions $v$. placebo on the proportion of healthy days over the 6-week study period). On each day, participants scored cold/flu SI $(0=$ not experiencing to $3=$ very intense or severe) for each of the following nine symptoms: running/congested nose; stiffness or chills; headache; cough; fatigue; fever; sore throat; achiness; ear discomfort. The cold/flu SI score was calculated daily and was the sum of the individual SI. A SI score $\leq 6$ was considered to be a healthy day, and a SI score $>6$ was designated as a day of cold/flu ${ }^{(1)}$.

\section{Secondary outcomes}

A day of cold/flu was designated as a new episode if there was $\geq 1$ healthy day between days of cold, and duration was defined as the average number of days of cold/flu per episode per subject. Participant characteristics and level of academic stress over time were also examined to determine which characteristics were informative for the proportion of participants who were likely to report a cold on any given day. Salivary sIgA was measured as a potential biomarker of cold/flu. Participants were asked to come to the study site on any day during week 0 and then, again, during the week of final exams between 15.30 and 18.30 hours to provide a saliva sample. Investigators confirmed that participants had not brushed or flossed their teeth $60 \mathrm{~min}$ before saliva collection. Stimulated collections were obtained over 1 min using a cotton swab with citric acid preparation and a collection tube (Salivette; Sarstedt, Inc.). The saliva samples were placed on ice until centrifuged for $2 \mathrm{~min}$ at $1000 \mathrm{~g}$. The samples were frozen at $-70^{\circ} \mathrm{C}$ until assayed for SIgA via ELISA (ALPCO Immunoassays). Salivary SIgA concentrations were not measured if a participant provided only one of the two samples.

\section{Statistical analyses}

Based on a previous study ${ }^{(1)}$, which observed a $40 \%$ reduction in the percentage of days with a cold/flu over a period of 8 weeks using a prebiotic in the same model of academic stress, a sample size of 145 per group was estimated to be necessary to observe a $50 \%$ reduction in the proportion of days with cold symptoms with a SI score $>6$ ( $80 \%$ power, $P=0.05,10 \%$ attrition rate) when each supplement was compared individually with the placebo group over a 6-week period.

The following variables were analysed for intervention effects: the proportion of healthy days reported by each participant during the study period (primary outcome); the proportion of participants reporting at least $1 \mathrm{~d}$ with a cold/flu; the number of episodes of cold/flu reported by each participant during the study period; the duration of an episode. The proportion of healthy days and the proportion of participants reporting at least $1 \mathrm{~d}$ with cold/flu symptoms were analysed using logistic ANOVA with an overdispersion scale parameter to capture the variance being larger than expected under a binomial model due to possible clustering of days with a cold. The estimated overdispersion parameter varied among the interventions; thus, an overdispersed logistic ANOVA comparing each intervention against the placebo was also run. The number and duration of episodes of cold/flu symptoms during the study period were analysed using Poisson ANOVA.

Data on the proportion of participants reporting a cold/flu on any given day were analysed according to a generalised linear mixed model using a binary distribution where the probability of having cold/flu symptoms on a given day is a function of the fixed effects of intervention code, week of study (excluding week 0), sex, hours of sleep the previous night, self-reported stress score, indicator of 2012-13 flu vaccine, indicator of 2011-12 flu vaccine, BMI at baseline and SI score category during week 0 . The SI score category during week 0 was based on the average sum of SI for the nine cold/flu symptoms during week 0 . Participants were categorised as having an average sum of SI of $<1$ (61\% of the participants), $1-3$ (26\% of the participants) or $>3(13 \%$ of the participants) during week 0 to control for participant variation in reporting symptoms. Interactions of each variable with intervention code were included. Hours of sleep, stress score and BMI were treated as continuous variables; all the others were assumed to be categorical variables. To account for the daily repeated observations, an autoregressive covariance matrix for the residuals was included to capture the correlation of adjacent daily observations for each participant. A full model with all fixed effects and two-way interactions of the effects with intervention code and with the autoregressive covariance was fitted to the daily data. Interactions and main effects (other than intervention) were removed hierarchically, starting with the interaction effect with the largest $P$ value, until only significant effects or effects with hypotheses remained in the model. Intervention and intervention $\times$ week interactions were retained in the model regardless of significance. Comparison of intervention means was made at several sets of values for the continuous effects if one or more of those were found to be significant and if they interacted with intervention. If they did not interact but were significant, then intervention means were calculated for the 
average values of the significant continuous effects. The Kenward-Roger method was used to adjust for bias in the estimators due to the non-diagonal covariance matrix; the Tukey-Kramer method was used to control for the experiment-wise error rate. All tests were conducted using a family-wise type I error rate of $\alpha=0 \cdot 05$.

On the first day of the study (before beginning the study procedures), three participants were excluded because they reported being sick. Additionally, eleven participants were excluded because they reported taking antibiotics during the period between obtaining informed consent and the start of the study. Furthermore, twenty-four participants reported a SI score on their daily questionnaire that classified them as having a cold, and four participants reported taking an antibiotic on the 1st day of the study. When these twenty-eight participants were excluded from the dataset and the primary outcome was re-examined, the results were unchanged. All statistical analyses were performed using SAS version 9.2 (SAS Institute). Simple tests at baseline and plots were executed with the use of SigmaPlot for Windows (Build 12.1.0.15 version 12.0, 2011; Systat Software, Inc.). Data were analysed on an intent-to-treat basis and are presented as least-squares means unless otherwise stated.

\section{Results}

\section{Participants and compliance}

Initially, 921 students were screened for eligibility, of which 655 consented and 583 were randomised to one of the four interventions (Fig. 1). The majority of the participants were female, non-Hispanic, white students with a BMI reflective of normal weight. On average, participants reported taking $>90 \%$ of the supplement capsules (Table 1). Mid-way through the study, two participants were withdrawn by the principal investigator because they did not complete any daily questionnaires post-randomisation and were then lost to follow-up. Of the total study participants, 581 were included in the analyses. Over the course of the study, three participants withdrew: the first participant withdrew after taking $L$. helveticus for $7 \mathrm{~d}$ due to a loss of interest in participating; the second withdrew after $1 \mathrm{~d}$ on $B$. infantis due to gastrointestinal discomfort; the third withdrew after taking the placebo for $25 \mathrm{~d}$ due to abdominal pain (Fig. 1). Throughout the study, one participant complained of increased appetite (placebo) and another of a 'popping knee' while exercising (L. helveticus). After approximately 2 weeks of supplementation, two participants discontinued the supplement (placebo and B. bifidum) due to diarrhoea, but completed the remainder of the study-related activities.

\section{Primary outcome}

Proportion of healthy days. A total of 177 participants reported $701 \mathrm{~d}$ of cold/flu (placebo, $222 \mathrm{~d}$; L. helveticus $222 \mathrm{~d}$; B. infantis $139 \mathrm{~d}$; B. bifidum $118 \mathrm{~d}$ ) during the 6-week intervention period. When the proportion of healthy days for each intervention was compared with placebo (Table 2), a greater proportion of healthy days was observed with B. bifidum $(P=0.05)$, but not with $B$. infantis $(P=0 \cdot 12)$ or L. helveticus $(P=0 \cdot 96)$.

\section{Secondary outcomes}

The percentage of participants who reported $\geq 1 \mathrm{~d}$ of cold/flu during the 6-week intervention period was significantly lower with $B$. bifidum than with placebo $(P<0.05$; Table 2$)$. This was not observed with the other two interventions. There were also fewer episodes of cold/flu with $B$. bifidum $(P<0.05)$ and a trend towards fewer episodes with $B$. infantis $(P=0.07)$ compared with placebo. Although there was

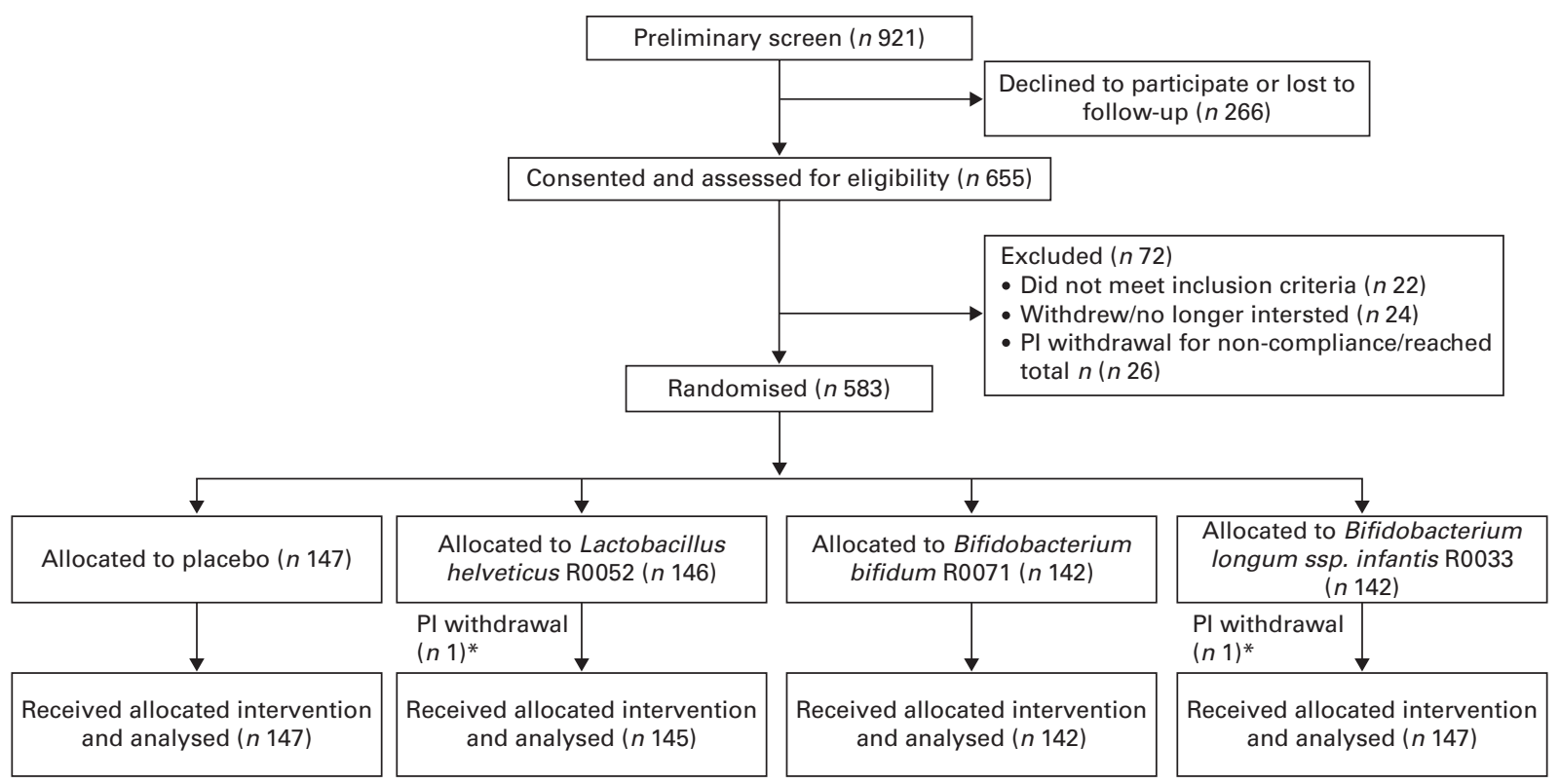

Fig. 1. Participant flow throughout the study. *Participants were withdrawn by the principal investigator (PI) because daily questionnaires were not completed. 
Table 1. Participant characteristics and compliance during the 6-week intervention period by group (Least-squares means (LS-mean) with their standard errors)

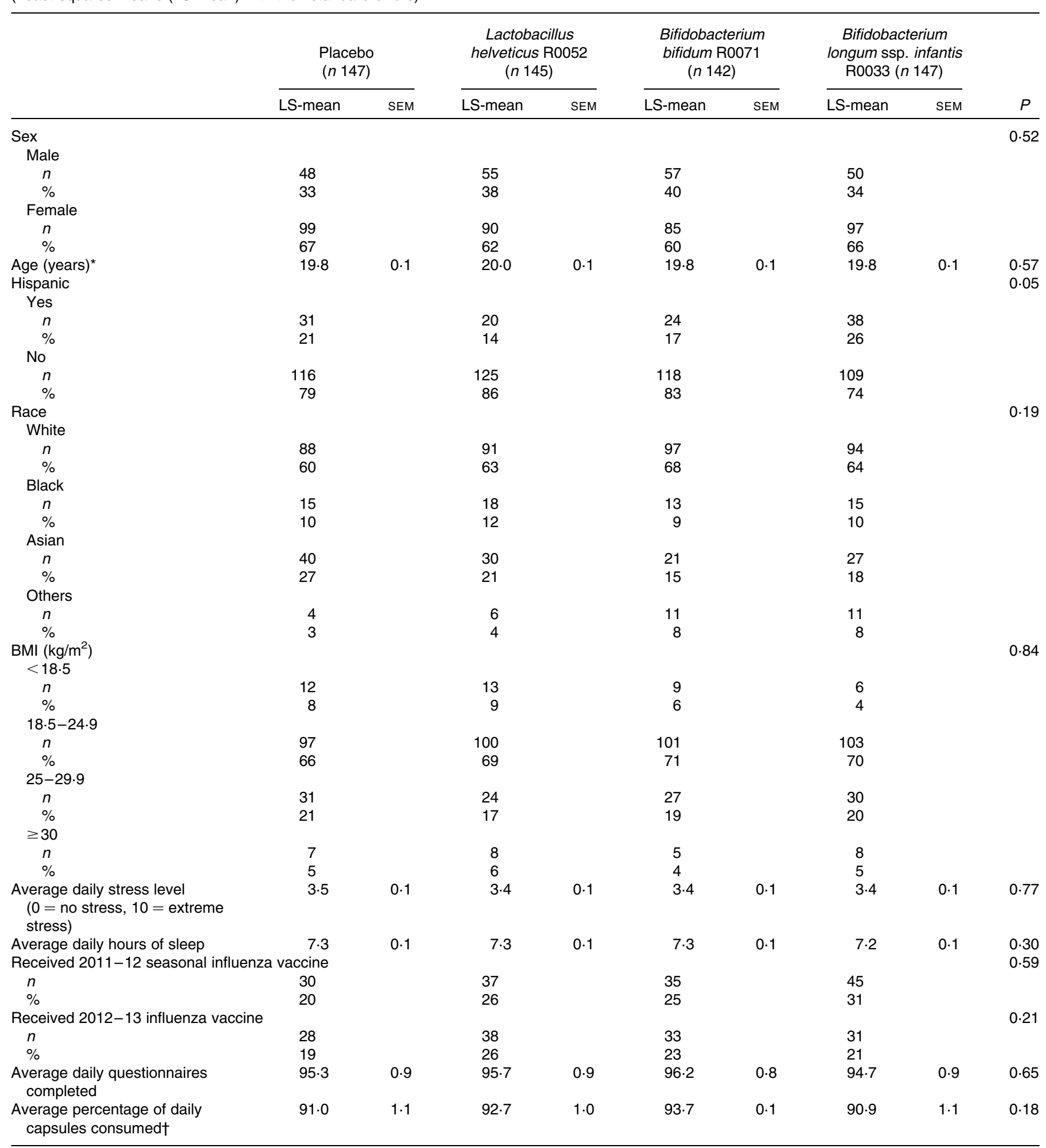

${ }^{*} \chi^{2}$ and ANOVA tests.

† If a daily questionnaire was not returned on a day, it was assumed that the study capsule was not consumed.

an overall effect of the interventions on the duration of cold/flu episodes $(P<0.05)$, this effect was due to a trend $(P=0.07)$ in the difference between the duration of cold/flu with $B$. bifidum and $L$. helveticus (Table 2). There was no difference in the duration of cold/flu between placebo and any of the other interventions.

A predictive model was used to determine what characteristics were informative for the proportion of participants who 
Table 2. Characteristics of cold/flu days and salivary secretory $\lg A(\lg A)$ by group during the 6-week intervention period (Least-squares means (LS-mean) with their standard errors)

\begin{tabular}{|c|c|c|c|c|c|c|c|c|c|}
\hline & \multicolumn{2}{|c|}{$\begin{array}{l}\text { Placebo } \\
(n 147)\end{array}$} & \multicolumn{2}{|c|}{$\begin{array}{c}\text { Lactobacillus } \\
\text { helveticus R0052 } \\
(n 145)\end{array}$} & \multicolumn{2}{|c|}{$\begin{array}{l}\text { Bifidobacterium } \\
\text { bifidum R0071 } \\
\text { ( } n \text { 142) }\end{array}$} & \multicolumn{2}{|c|}{$\begin{array}{l}\text { Bifidobacterium } \\
\text { longum ssp. infantis } \\
\text { R0033 (n 147) }\end{array}$} & \multirow[b]{2}{*}{$P$} \\
\hline & LS-mean & $\overline{\text { SEM }}$ & LS-mean & $\overline{\text { SEM }}$ & LS-mean & $\overline{\text { SEM }}$ & LS-mean & $\overline{\text { SEM }}$ & \\
\hline $\begin{array}{l}\text { Proportion of healthy days } \| \\
\text { Participants with } \geq 1 \mathrm{~d} \text { of cold/fluq }\end{array}$ & 0.96 & 0.01 & 0.96 & 0.01 & $0.98^{*}$ & 0.01 & 0.98 & 0.01 & 0.104 \\
\hline $\begin{array}{l}n \\
\%\end{array}$ & $\begin{array}{l}55 \\
37\end{array}$ & & $\begin{array}{l}43 \\
30\end{array}$ & & $\begin{array}{c}34 \\
24^{*}\end{array}$ & & $\begin{array}{l}45 \\
31\end{array}$ & & \\
\hline Episodes of cold/flu $u^{\star *}$ & 0.63 & 0.07 & 0.61 & 0.07 & $0.43^{*}$ & 0.06 & $0.48 \dagger$ & 0.06 & 0.046 \\
\hline Duration of cold/flu†† & $2 \cdot 4$ & 0.2 & $2 \cdot 7$ & 0.2 & $1.8 \ddagger$ & 0.2 & $2 \cdot 0$ & 0.2 & 0.039 \\
\hline Salivary slgA, $n \ddagger \ddagger$ & 119 & & 120 & & 110 & & 125 & & G: 0.223 \\
\hline Baseline $(\mu \mathrm{g} / \mathrm{ml})$ & 148 & 11 & 135 & 9 & 131 & 10 & 147 & 11 & $\mathrm{D}:<0.001$ \\
\hline Final $(\mu \mathrm{g} / \mathrm{ml})$ & $166 \S$ & 11 & $167 \S$ & 10 & $138 \S$ & 10 & 157 & 13 & $\mathrm{G} \times \mathrm{D}: 0.232$ \\
\hline
\end{tabular}

$D$, indicator of illness on the reported day; $G$, intervention group.

* LS-mean or proportion was significantly different from that of placebo $(P \leq 0.05)$.

t LS-mean tended to be different from that of placebo $(P=0.07)$.

†LS-mean tended to be different from that of $L$. helveticus $(P=0.07)$.

$\S$ LS-mean was significantly different from that at baseline $(P<0.05)$.

$\|$ A healthy day was defined as a day with a cold/flu symptom intensity score $\leq 6$ (logistic ANOVA).

I A day of cold/flu was defined as a day where the sum of symptom intensities (scored $0=$ no symptom to $3=$ severe intensity of symptom) for each of the nine cold/flu symptoms was $>6$ (logistic ANOVA).

** A day of cold/flu was designated as a new episode if there was $\geq 1 \mathrm{~d}$ between with no cold (Poisson ANOVA).

†† Duration was defined as the average number of days of cold/flu per episode per subject (Poisson ANOVA).

$\ddagger \ddagger$ Data were analysed using a log transformation and are reported as means (generalised linear mixed model). Salivary slgA concentrations were not measured if a participant provided only one of the two samples.

were likely to report a cold on any given day during the study period. In this model, the intervention $(P<0.05)$, week of study $(P<0 \cdot 01)$, sex $(P<0 \cdot 01)$, and the interaction between intervention and stress $(P<0.05)$, sleep $(P<0.05)$ and SI score category during week $0(P<0 \cdot 01)$ were predictive of reporting a cold/flu on any given day. Receiving the annual flu vaccine for the current or previous years and BMI were not predictive, and were thus dropped from the model. The proportion of participants who reported a cold on any given day was lower at weeks 2 and 3 with $B$. bifidum and $B$. infantis than with placebo and $B$. infantis at week $3 v$. week 1 (Fig. 2) for an average level of stress (i.e. 3) and the most commonly reported number of hours of sleep $(8 \mathrm{~h})$. The proportion of female participants (0.032 (SEM 0.003)) who reported a cold on any given day was greater than that of males $(0.019$ (SEM 0.003), $P<0.01)$. The relationship between stress and the probability of reporting cold/flu symptoms varied by intervention and was always positive; furthermore, all the four slopes were significantly different from 0. The placebo group had the smallest slope (0.0765, $P<0.05)$, and $L$. helveticus had a slope of $0.1316(P<0.01)$. $B$. infantis had a slope slightly larger than $L$. belveticus $(0 \cdot 15, \quad P<0 \cdot 01)$, and $B$. bifidum had the highest slope (0.2537, $P<0.01)$; however, the overall estimated proportion of participants reporting a cold on any given day was still lower with $B$. infantis and B. bifidum than with placebo at almost all levels of stress. In order to see the relationships between the probability of reporting a day of cold/flu and stress by intervention and sex, the probability of reporting a cold for females and males in week 4 who sleep $8 \mathrm{~h} /$ night and who had a SI score during week 0 that averaged between 1 and 3 was plotted over the range of possible daily stress values (Fig. 3). Other combinations of factors have similar behaviour but with different intercepts. The relationship between hours of sleep and the probability of reporting a cold/flu on any given day was significant only for B. infantis, where it was found that the slope on a linear scale was $-0 \cdot 143$ $(P<0 \cdot 01)$. Hence, for $B$. infantis only, the probability of

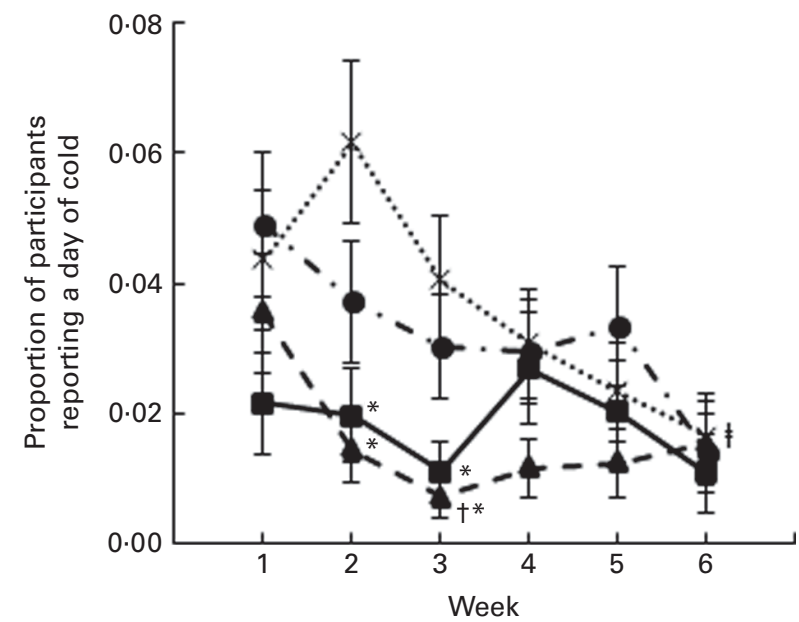

Fig. 2. Proportion of participants who were likely to report a cold/flu for any given day as a function of week $(P<0.01)$ and intervention $(P<0.05)$. Values are least-squares means, with their standard errors represented by vertical bars, and are based on averaging over all the other factors in the model (sex $(P<0.01)$, stress $(P<0.05$ for stress $\times$ intervention), sleep $(P<0.05$ for sleep $\times$ intervention) and symptom intensity category $(P<0.01$ for symptom intensity category $\times$ intervention)) and adjusted to a level of stress equal to 3 and $8 \mathrm{~h}$ sleep. * Least-squares mean was significantly different from that of placebo $(* x)$ at the same week $(P<0.05)$. †Least-squares mean was significantly different from that at week $1(P<0.01)$. łLeast-squares mean was significantly different from that at week $2(P<0.05)$ (generalised linear mixed model). ---, Lactobacillus helveticus R0052; _- , Bifidobacterium bifidum R0071; - ^-, Bifidobacterium longum ssp. infantis R0033. 
(a)

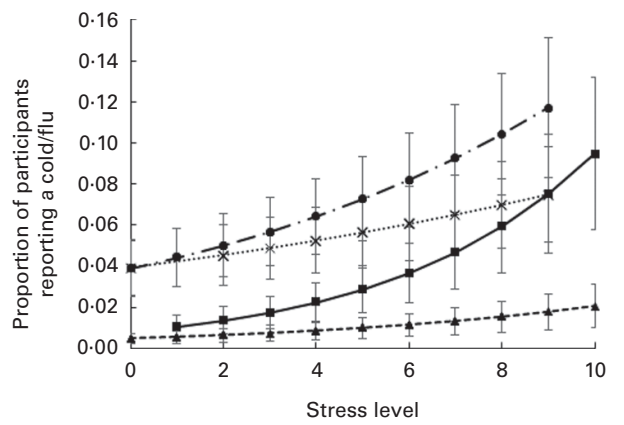

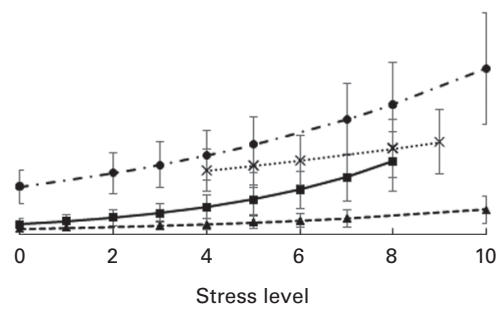

Fig. 3. Proportion of participants who were likely to report a day of cold/flu for any given day as a function of intervention and stress level $(P<0.05$ for intervention $\times$ stress level; $0=$ no stress, $10=$ extremely stressed) for (a) females and (b) males in week 4 of the study and who had a symptom intensity score during week 0 that averaged between 1 and 3 and who slept $8 \mathrm{~h}$. Values are least-squares means, with their standard errors represented by vertical bars. $P<0.05$ for females compared with males (generalised linear mixed model). ......., Placebo; ---, Lactobacillus helveticus R0052; -n-, Bifidobacterium bifidum R0071; - -, Bifidobacterium longum ssp. infantis R0033.

reporting a day of cold/flu decreased as the hours of sleep increased. There was no significant relationship between hours of sleep and the probability of reporting a day of cold for the other three interventions, i.e. the slopes were not statistically different from 0 ( $L$. helveticus slope $=0 \cdot 045, P=0 \cdot 20$; $B$. bifidum slope $=-0.0045, \quad P=0.9224 ;$ placebo slope $=$ $-0.0375, \quad P=0.25)$. The proportion of participants who reported a SI score of $1-3$ during week 0 and then received $B$. bifidum or $B$. infantis was less likely to report a cold on any given day during the intervention than those who received $L$. helveticus or placebo ( $P<0 \cdot 01$, data not shown).

A similar model was used to examine the average daily SI score across all 6 weeks of the intervention. In this model, sex $(P<0.01)$ and the interaction between intervention and week $(P<0.01$; Fig. 4$)$, stress $(P<0 \cdot 01)$ and the interaction between stress and intervention $(P<0 \cdot 01)$, and SI score category during week $0(P<0 \cdot 01)$ and the interaction between SI score category and intervention $(P<0.01)$ were predictive for the daily SI score. On average, females reported a higher daily SI score (1.19 (SEM 0.06)) than males (0.65 (SEM 0.07)). At a stress level of 3 and $8 \mathrm{~h}$ sleep, participants receiving $B$. infantis reported a lower daily SI score for week 2 than those receiving placebo $(P<0 \cdot 05$; Fig. 4). The relationship between stress and the daily SI score was positive; however, $B$. bifidum was the only group with a higher slope than placebo (data not shown). The categorised average daily SI score at week 0 was predictive of the average daily SI score throughout the intervention. Participants who reported an average daily SI score of $<1$ during week 0 reported a lower average SI score during the intervention compared with those who reported a score of $1-3$ or $>3$ during week 0 . This held true for all interventions except $B$. infantis. Participants who took $B$. infantis and reported an average daily SI score of 1-3 during week 0 did not report a score that was different from that reported by participants with a score of $<1$ at week 0 (results not shown).

The concentration of salivary SIgA was not different at baseline between participants who never reported a day of cold/flu and those who went on to report $\geq 1 \mathrm{~d}$ of cold/flu during the 6-week intervention period (no cold: 142 (SEM 7) $\mu \mathrm{g} / \mathrm{ml}, n$ 328; $\geq 1 \mathrm{~d}$ of cold/flu: 136 (sem 8) $\mu \mathrm{g} / \mathrm{ml}, n$ 146).
The concentration of salivary sIgA increased between the measurement taken at baseline and that obtained during final exams $(P<0 \cdot 01$; Table 2$)$ for all interventions except $B$. infantis. However, no differences between placebo and any of the three investigational supplements were observed.

\section{Discussion}

The primary aim of the present study was to examine the effect of three different potentially probiotic bacteria on the proportion of healthy days over a 6 -week period during which undergraduate students underwent academic stress. There was a greater proportion of healthy days with $B$. bifidum than with placebo but not with $B$. infantis or $L$. helveticus. A previous study that used this academic stress model and defined days of cold/flu using the same criteria observed a $40 \%$ reduction in the percentage of days with a cold/flu over an 8 -week period



Fig. 4. Average daily symptom intensity (SI) score during the 6-week intervention period $(P<0.01$ for week $\times$ intervention). Values are least-squares means, with their standard errors represented by vertical bars, and are based on averaging over all the other factors in the model (sex $(P<0.01)$, stress $(P<0.01$ for stress $\times$ intervention) and SI category $(P<0.01$ for $\mathrm{SI}$ category $\times$ intervention)) and adjusted to a level of stress equal to 3 and $8 \mathrm{~h}$ sleep. *Least-squares mean was significantly different from that of placebo $(\ldots \times \ldots)$ at week 2 (general linear mixed model). -๑--, Lactobacillus helveticus R0052; -ـ-, Bifidobacterium bifidum R0071; -^-, Bifidobacterium longum ssp. infantis R0033. 
with the supplementation of $5 \mathrm{~g}$ GOS$/ \mathrm{d}^{(1)}$. However, during that academic semester, cold/flu was observed on approximately $10 \%$ of the days, whereas in the present study, cold/flu was observed on only $4 \%$ of the days with placebo and $2 \%$ of the days with $B$. infantis. It is possible that the lower percentage of days with a cold/flu made it more difficult to achieve statistical significance with $B$. infantis or L. helveticus. Additionally, probiotic-induced cytokine production by epithelial and immune cells varies by genus and species ${ }^{(6,10)}$, suggesting that immune responses and health outcomes would also differ.

As part of the secondary outcomes, the percentage of participants who reported $\geq 1 \mathrm{~d}$ of cold/flu, the number of episodes and the duration of each episode were examined. The percentage of participants who had at least one episode of cold/flu and the total number of episodes was lower with $B$. bifidum. There was also a trend towards a shorter duration per episode with $B$. bifidum. There were no significant effects of $B$. infantis or $L$. belveticus for these outcomes. These data are different from those reported by King et al. ${ }^{(6)}$ who found a shorter episode duration with Lactobacillus and Bifidobacterium strains, in general, than with placebo when used alone or more often in combination with other probiotics. These differences may also be due to how an episode or day of cold/flu is defined. de Vrese et $a l^{(3)}$ defined an episode as having at least one specific symptom of a respiratory tract infection. Rizzardini et al. ${ }^{(15)}$ defined an episode as having a fever plus one additional symptom of influenza. At least three symptoms were required in the present study to designate an episode. The definition of when one episode of cold/flu ends and another begins is also different among studies or may even be left undefined. This ambiguous factor may have an impact on the average duration. King et al. ${ }^{(6)}$ defined the duration of illness episodes as the 'overall sum of illness episode lengths (in days) divided by the total number of illness episodes experienced by the study participants'. Using this method, an individual who has an extended illness could inflate the average duration. Also, this method does not control for multiple episodes in a single participant. To minimise these influences, the present study averaged the mean duration of cold/flu for each participant who reported $\geq 1 \mathrm{~d}$ of cold/flu.

Participant characteristics and the level of academic stress were included in a predictive model to determine what factors were informative for the proportion of participants who were likely to report a cold on any given day. The interaction between intervention and level of stress, hours of sleep and SI score category during week 0 was significant. Sex and week of the study intervention also influenced the likelihood of reporting a cold on any given day. By including these influential factors in the model, a significant effect of both strains of bifidobacteria was observed, with a lower proportion of participants reporting a cold on any given day at weeks 2 and 3 with $B$. bifidum and B. infantis than with placebo (Fig. 2). As anticipated, an increase in the level of stress resulted in an increase in the proportion of participants who were likely to report a cold/flu on any given day ${ }^{(1)}$; however, the slope of this increase was greatest with bifidobacteria (Fig. 3). These results suggest that these strains of bifidobacteria were protective at lower levels of stress, but there was less of a protective effect at higher levels of stress; regardless, the overall estimated proportion of participants reporting a cold on any given day was still lower with bifidobacteria than with placebo at almost all levels of stress. For the interaction between intervention and hours of sleep, the probability of reporting a cold/flu on any given day decreased with $B$. infantis as hours of sleep increased. The loss of the protective effects with higher levels of stress or less sleep is not surprising, given that probiotics are dietary supplements that help modulate immune responses in the context of an overall healthy lifestyle.

The SI score category during week 0 was included in the model because it was previously noted that some participants routinely report higher scores for cold/flu symptoms. These participants were more likely to report a cold/flu because their average SI score tended to be higher ${ }^{(1)}$. By including this and its interaction with intervention, the effect of the interventions could be more fully explored. In the present study, there was a statistically significant interaction between intervention and SI score category where the proportion of participants who reported a SI score of $1-3$ during week 0 and then received $B$. bifidum or B. infantis was less likely to report a cold on any given day during the intervention than those who received $L$. helveticus or placebo $(P<0 \cdot 01$, data not shown). This may suggest that for participants who routinely reported lower scores (SI score 1-3), bifidobacteria prevented some cold/flu symptoms such that the total SI score was never $>6$, thereby denoting a day of cold/flu. This was not the case when participants received a placebo or the $L$. helveticus intervention.

The proportion of females who reported a cold on any given day was greater than that of males. This is in agreement with Matheson et al. ${ }^{(13)}$ who showed that adult women develop more non-atopic as well as atopic rhinitis than males. However, females also had a higher average daily SI score during the 6-week intervention period, suggesting that there may be a difference between sexes in how they report symptoms or that women actually experienced more cold/flu symptoms than men. Others have shown that women self-report more infectious illness symptoms during the perimenstrual period ${ }^{(12)}$. It is possible that menstrual cycle-related diarrhoea and distress contributed to the overall level of stress and ultimately days of cold/flu ${ }^{(12)}$. Interestingly, females did report a higher level of stress than males across the study period (data not shown). Future studies should include sex in the statistical model to control for some of the unexplained variability in the intervention effect.

sIgA is the main Ig secreted by immune cells within mucosal tissues and serves as a first line of defence against infection. Gleeson et $a l .{ }^{(16)}$ reported that salivary SIgA concentrations were maintained in athletes taking $L$. casei Shirota but not in those taking placebo, and that the number of self-reported episodes of the upper respiratory tract infection was lower with the probiotic. Interestingly, 50 of the $65(77 \%)$ participants reported at least one episode of cold/flu over the 16-week study period. In the present study, no differences in sIgA concentrations were observed between any of the probiotics and placebo; however, an increase in sIgA concentrations was observed between 
baseline and the week of final exams with the supplementation of placebo, L. helveticus and B. bifidum. Previously, Kiecolt-Glaser et $a l .{ }^{(17)}$ observed an increase in plasma IgA concentrations but no change in salivary IgA concentrations in medical students on the 1st day of exams compared with a non-exam period of the academic year. Others have shown an acute increase in salivary sIgA concentrations during mental $\operatorname{arithmetic}^{(18)}$ and a decrease in concentrations 2 weeks following major academic exams ${ }^{(19)}$. It may be possible that any probiotic-mediated increases in salivary SIgA concentrations were obscured by stress-mediated changes.

In conclusion, daily intake of $B$. bifidum resulted in a greater proportion of healthy days and a lower percentage of participants reporting $\geq 1 \mathrm{~d}$ of cold/flu during a 6-week intervention period surrounding and including the time of academic exams. Daily intake of B. bifidum and B. infantis resulted in a lower proportion of participants reporting a cold on any given day at weeks 2 and 3 than that of placebo when level of stress, sex, week of study, hours of sleep and SI score category during week 0 were included in the model. These effects were not observed with L. helveticus, suggesting that the effect of the probiotics on self-reported cold/flu symptoms is genus and species specific.

\section{Acknowledgements}

The authors thank Thomas Tompkins and Ronan Gire from Lallemand Health Solutions, as well as Abdulah Hanifi, Tyler Culpepper and the undergraduate students from the University of Florida who helped with various aspects of this study.

The present study was funded by Lallemand Health Solutions, the study sponsor, and the University of Florida Agriculture Experiment Station. They also provided the study supplements, but otherwise did not contribute to the design, implementation or interpretation of the data.

The authors' responsibilities were as follows: B. L.-H., M. C. C., C. N. and W. J. D. designed the research; B. L.-H., C. C. R., A. L. F., C. N., L. K., G. J. S. and S. J. S. conducted the research; M. C. C. analysed the data; B. L.-H., C. C. R., A. L. F., S.-A. G. and M. C. C. wrote the manuscript; B. L.-H. had primary responsibility for the final content. All the authors read and approved the final manuscript.

B. L.-H., M. C. C. and W. J. D. received research support or contract funding from the study sponsor for other projects. At the time of the study, S.-A. G. was employed by the study sponsor. None of the other authors had a personal or financial conflict of interest.

\section{References}

1. Hughes C, Davoodi-Semiromi Y, Colee JC, et al. (2011) Galactooligosaccharide supplementation reduces stressinduced gastrointestinal dysfunction and days of cold or flu: a randomized, double-blind, controlled trial in healthy university students. Am J Clin Nutr 93, 1305-1311.

2. Kiecolt-Glaser JK, Glaser R, Strain EC, et al. (1986) Modulation of cellular immunity in medical students. J Behav Med 9, 5-21.
3. de Vrese M, Winkler P, Rautenberg P, et al. (2006) Probiotic bacteria reduced duration and severity but not the incidence of common cold episodes in a double blind, randomized, controlled trial. Vaccine 24, 6670-6674.

4. Vulevic J, Drakoularakou A, Yaqoob P, et al. (2008) Modulation of the fecal microflora profile and immune function by a novel trans-galactooligosaccharide mixture (B-GOS) in healthy elderly volunteers. Am J Clin Nutr 88, 1438-1446.

5. Davis LM, Martinez I, Walter J, et al. (2011) Barcoded pyrosequencing reveals that consumption of galactooligosaccharides results in a highly specific bifidogenic response in humans. PLOS ONE 6, e25200.

6. King S, Glanville J, Sanders ME, et al. (2014) Effectiveness of probiotics on the duration of illness in healthy children and adults who develop common acute respiratory infectious conditions: a systematic review and meta-analysis. Br J Nutr 112, 41-54.

7. Segerstrom SC \& Miller GE (2004) Psychological stress and the human immune system: a meta-analytic study of 30 years of inquiry. Psychol Bull 130, 601-630.

8. Smith TJ, Rigassio-Radler D, Denmark R, et al. (2013) Effect of Lactobacillus rhamnosus LGG(R) and Bifidobacterium animalis ssp. lactis BB-12(R) on health-related quality of life in college students affected by upper respiratory infections. Br J Nutr 109, 1999-2007.

9. Timmerman HM, Koning CJ, Mulder L, et al. (2004) Monostrain, multistrain and multispecies probiotics - a comparison of functionality and efficacy. Int J Food Microbiol 96, 219-233.

10. Ashraf R \& Shah NP (2014) Immune system stimulation by probiotic microorganisms. Crit Rev Food Sci Nutr $\mathbf{5 4}$, 938-956.

11. Cazzola M, Pham-Thi N, Kerihuel JC, et al. (2010) Efficacy of a synbiotic supplementation in the prevention of common winter diseases in children: a randomized, double-blind, placebo-controlled pilot study. Ther Adv Respir Dis 4, 271-278.

12. Groer M, Carr J \& Younger MS (1993) Relationships between self-reported symptoms of infection, menstrual-cycle-related distress, and cycle phase. Behav Med 19, 13-19.

13. Matheson MC, Dharmage SC, Abramson MJ, et al. (2011) Early-life risk factors and incidence of rhinitis: results from the European Community Respiratory Health Study - an international population-based cohort study. e815J Allergy Clin Immunol 128, 816-823. e815.

14. Langkamp-Henken B (2012) Assessing immune health outcomes following dietary interventions in healthy adults - a model using galactooligosaccharides. In Dietary Fiber and Health, pp. 157-168 [SS Cho and N Almeida, editors]. Boca Raton: CRC Press.

15. Rizzardini G, Eskesen D, Calder PC, et al. (2012) Evaluation of the immune benefits of two probiotic strains Bifidobacterium animalis ssp. lactis, BB-12(R) and Lactobacillus paracasei ssp. paracasei, L. casei 431(R) in an influenza vaccination model: a randomised, double-blind, placebocontrolled study. Br J Nutr 107, 876-884.

16. Gleeson M, Bishop NC, Oliveira M, et al. (2011) Daily probiotic's (Lactobacillus casei Shirota) reduction of infection incidence in athletes. Int J Sport Nutr Exerc Metab 21, 55-64.

17. Kiecolt-Glaser JK, Garner W, Speicher C, et al. (1984) Psychosocial modifiers of immunocompetence in medical students. Psychosom Med 46, 7-14.

18. Ring C, Drayson M, Walkey DG, et al. (2002) Secretory immunoglobulin A reactions to prolonged mental arithmetic stress: inter-session and intra-session reliability. Biol Psychol 59, 1-13.

19. Deinzer R, Kleineidam C, Stiller-Winkler R, et al. (2000) Prolonged reduction of salivary immunoglobulin A (sIgA) after a major academic exam. Int J Psychophysiol 37, 219-232. 